\title{
Application of 3D and 2D Imaging Techniques in the Examination of Suspect Tablets for the Detection of Counterfeit FDA-Regulated Products
}

\author{
Nicola Ranieri ${ }^{1}$, S. Frank Platek ${ }^{1}$, Douglas Albright ${ }^{1}$, and JaCinta S. Batson ${ }^{1}$ \\ 1. US Food and Drug Administration - Forensic Chemistry Center, Cincinnati, Ohio, USA.
}

Global statistics from economic organizations reporting on counterfeit products, such as the World Health Organization and the Pharmaceutical Security Institute, show medicinal drug counterfeiting is on the rise, and is contributing to a rapidly growing underground economy in both developed and developing countries [1], and to an increase of "rogue websites" selling potentially dangerous drugs $[2,3]$. As the income derived from these unlicensed organizations around the globe rises, so, too, does the number of victims. It is likely that at some point in one's lifetime a counterfeit tablet may be consumed $[2,3]$.

The United States Food and Drug Administration's Forensic Chemistry Center (FCC) has been involved in the detection of counterfeited products since the early 1990s. FCC has a history of performing toolmark analyses on compressed tablets by various techniques. In the past 25 years, the focus of the work performed has been determining manufacturing methods (associated with sourcing) of counterfeit tablets, and categorizing their physical characteristics and chemical properties. While the FCC can perform tablet analysis using an array of analytical techniques, this paper emphasizes the analysis of the physical characteristics relating to the manufacturing of tablets, and using these properties in a comparison analysis. Tablet analyses for authenticity are performed using two dimensional (2D) image overlays, and three dimensional (3D) profilometry, difference analysis measurements of tablet forms, and surface roughness.

Additionally, FCC performs toolmark analyses on compressed tablets using basic forensic microscopic approaches, such as bright field light microscopy techniques including oblique lighting. This technique casts shadows further highlighting the features not seen in direct bright field illumination. It is most effective for imaging toolmarks created by the upper and lower punches of the tableting equipment. (FIG 1) A more detailed light microscopy 2D method was developed by FCC incorporating a required "dark room" environment with $360^{\circ}$ diffused oblique lighting which enhances toolmark details. (FIG 2) For examinations of tablet toolmarks produced by the tool, light microscopy is a very powerful technique. However, seeking to include measurements of a third dimension (depth) is not a straightforward method, and does not meet the criteria recommended by the guidelines of the international standard measurement group, International Bureau of Weights and Measures (BIMP) [4]. Measurement guidelines established by BIMP include instrumentation based on the technology of focusvariation for both profile based and area surface measurements, such as the InfiniteFocus Measurement system (IFM). A primary use of the IFM is to measure dimensional tolerance, which is ideal for 3D measurements (depth) of the debossing, scoring, and detailed toolmarks from the manufacturing process. (FIG 3) The toolmarks created by the punches are transferred onto the faces of the suspect tablets, which allows comparison of the measurements with toolmarks found on authentic tablet faces over the same region [5]. The ability of the IFM to collect high resolution measurements enables both examination and comparison of the unique details produced during the manufacturing process of the upper and lower punches. The IFM allows surface reconstruction, measurement and profilometry with high accuracy [6]. A noticeable advantage of the IFM's 3D imaging capability over 2D light 
microscopy is the ability to image at almost vertical imaging steps with remarkable repeatability and accuracy at high resolution ( $1 \mu \mathrm{m}$ or smaller step increments) displaying highly detailed debossing features permitting identification of counterfeit tablet physical characteristics.

\section{References:}

[1] WHO http://apps.who.int/medicinedocs/documents/s21518en/s21518en.pdf?ua=1

[2] http://www.psi-inc.org/incidentTrends.cfm

[3] Roger Bate, Phake: The Deadly World of Fake Drugs, The AEI Press, 2012, p. 29.

[4] http://www.bipm.org/en/about-us/

[5] Dale Natoli, Tablets, 3rd Edition, Vol. 3, Informa Healthcare USA, Inc., 2008, pp. 1-48.

[6] Hartmuth Schroettner et al, Microchimica Acta, Vol. 155, Issue 1, September 2006, pp 279-284.

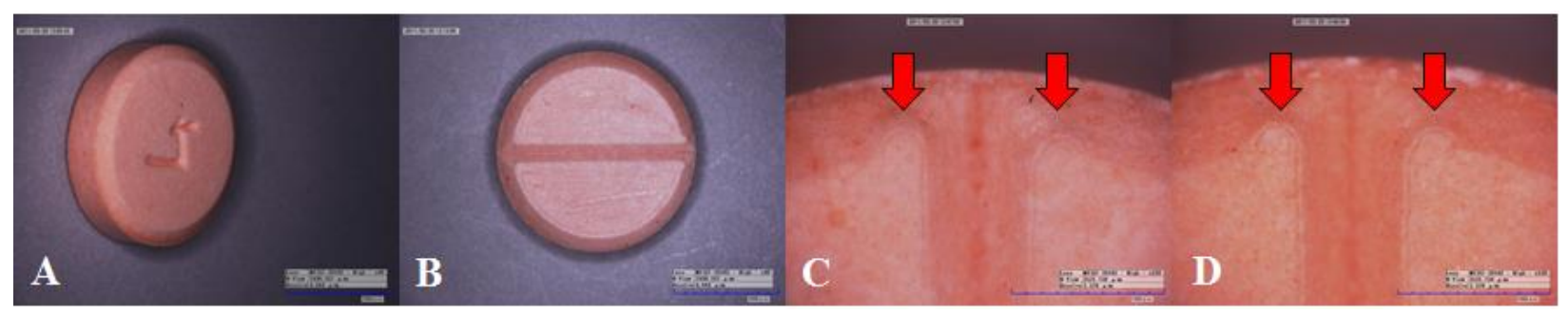

Figure 1. Oblique and bright field digital light microscopy (A \& B); toolmark (red arrows) of half score corners Tablet 1 (C) and Tablet 2 (D).
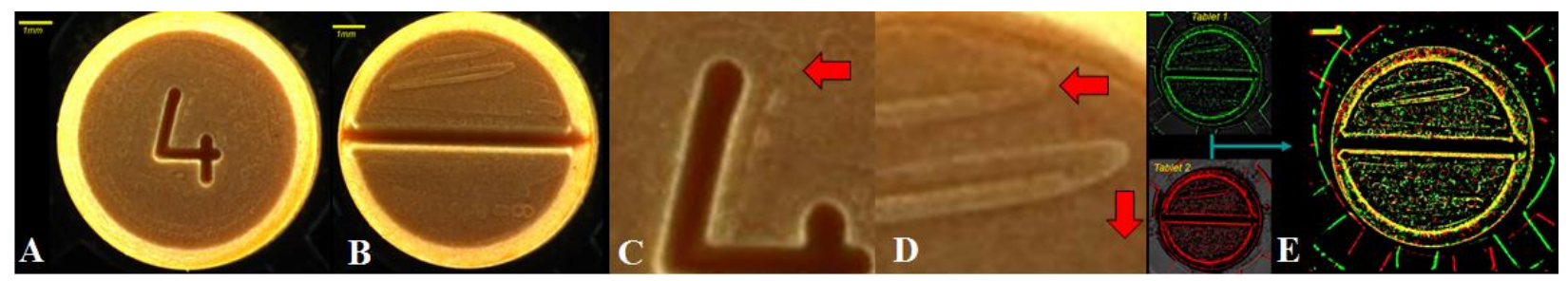

Figure 2. Diffused oblique light microscopy reveals more details than digital light microscopy (A \& B); close-up imaging shows enhanced toolmark details (red arrows) and color segmentation (C, D, \& E).
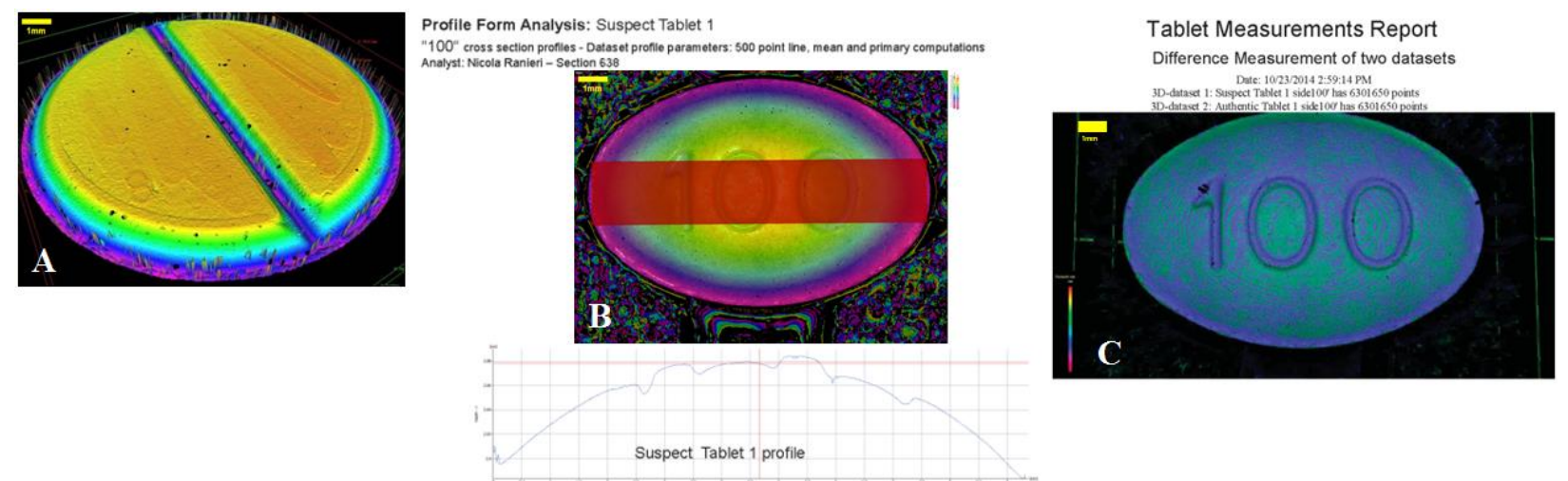

Figure 3. IFM 3D toolmarks visualization and ability to rotate the image at various viewing angles/orientations (A), profilometry (B), and difference measurements module (C). 